\title{
A Generic Meta-Model for a BIG ERP
}

\author{
Mustapha Hain ${ }^{1}$, Hicham Moutachaouik ${ }^{2}$, Abdelali Zakrani ${ }^{3}$ \\ ${ }^{123}$ (I2S2E Research Lab., SISM Team, Department of industry, \\ ENSAM, Hassan II University of Casablanca, Morocco)
}

\begin{abstract}
Enterprise Resource Planning (ERP) is business process management software that integrates all facets of an operation, including product planning, development, manufacturing, sales and marketing. The basic goal of using an ERP system is to provide one central repository for all information that is shared by all the various ERP facets to improve the flow of data across the organization. In contrast, the quality of making decision remains limited by the fact that this central repository is based the transactional and restricted data. In the paper, we propose to enhance this data by using the concept of the technologies Big Data (Using transactional and external data). The result of our proposition is a generic meta-model of an ERP rich which we call Big ERP. A meta-model proposed improves the quality of decisions made by the decisions-makers
\end{abstract}

Keywords: ERP; Big Data; decision-making; Meta-Model; decision process.

\section{Introduction}

ERP systems are integrated software solutions offered as off-the-shelf packages. ERP systems integrate information across the organization to manage organizational resources. There are several benefits of these systems: they include seamless information flow, provide access to real-time data, and enable processorientation and improved communication across the enterprise [1].

For enterprises, operating in the turbulent environment, directing towards the quality of decisionmaking processes, is becoming an essential component of management. The information support for decisionmaking processes at all levels of the enterprise, and the way of organizing them, is becoming increasingly important. Big data amounts not only to collecting data, but most of all, their processing and visualization, essential for obtaining business benefits [2].

To make decisions, an ERP system is based on the transactional and restricted data. Nevertheless, these decisions remain insufficient. That's why, this paper aims to improve the quality of making decision, enhanced insight and add value to the business by merging both data from Big Data and ERP. The paper is organized as follows. In Sect. 2, we briefly present the concepts of ERP, Big Data and related research that are relevant for this study. Sect. 3 presents our methodological approach. After that, we present, analyses and discusses our results in Sect. 4. Finally, we present our conclusions and implications for further research in Sect. 5.

\subsection{ERP}

\section{Erp, Big Data and Related Research}

[3] defined an ERP system as a "comprehensive, packet-based software solution that attempts to integrate all the processes and functions within a company to create a complete overview of the enterprise from a single IT architecture". [4] defined an ERP system as an information system that is designed to integrate and optimize business processes and transactions in a company.

The objective of an ERP system is to enhance the performance of the company by integrating all business processes and data into one system, including integration of the supply chain, inventory management, management of customers' orders, accounting, and human resource management $[5,6]$.

An ERP system is usually implemented in order to improve upon certain business goals, such as lowering inventory levels, reducing the working capital, shortening order lead times, or minimizing outstanding debts.

After the system went live, the shift managers were the only ones who knew how to use the system. Because they now had to spend their time entering data and completing business transactions, they had less time to attend to the production, stock keeping, and decision making.

The foundation of a generated system is an information model. Based on this model, the system components are created automatically. Depending on the underlying paradigm, an information model can describe business processes, operations, functions, objects, data, and/or organizational structures (Fig. 2).

\subsection{BIG DATA}

We view that several key themes with the Big Data trend include (i) using a cloud for large-scale external and internal data; (ii) providing an easy-to-use but powerful services to access/manage/analyze the Big Data in the cloud; (iii) defining a problem-solving space and developing an architecture for a Big Data 
environment to conceptualize goals, tasks, and problem-solving methods to apply to domains; and (iv) managing Big Data and analyzing them to discover business values [7].

Today, technology has turned the average consumer into an incessant generator of both traditional, structured, transactional data as well as more contemporary, unstructured, behavioral data. The magnitude of the data generated, there relentless rapidity at which data are constantly generated, and the diverse richness of the data are transforming marketing decision making. These three dimensions help define Big Data, commonly referred to as the three Vs: volume, velocity, and variety $[8,9,10]$. This model can be even extended to $5 \mathrm{~V}$ if the concepts of Veracity and value are incorporated into the Big Data definition (Fig. 1).

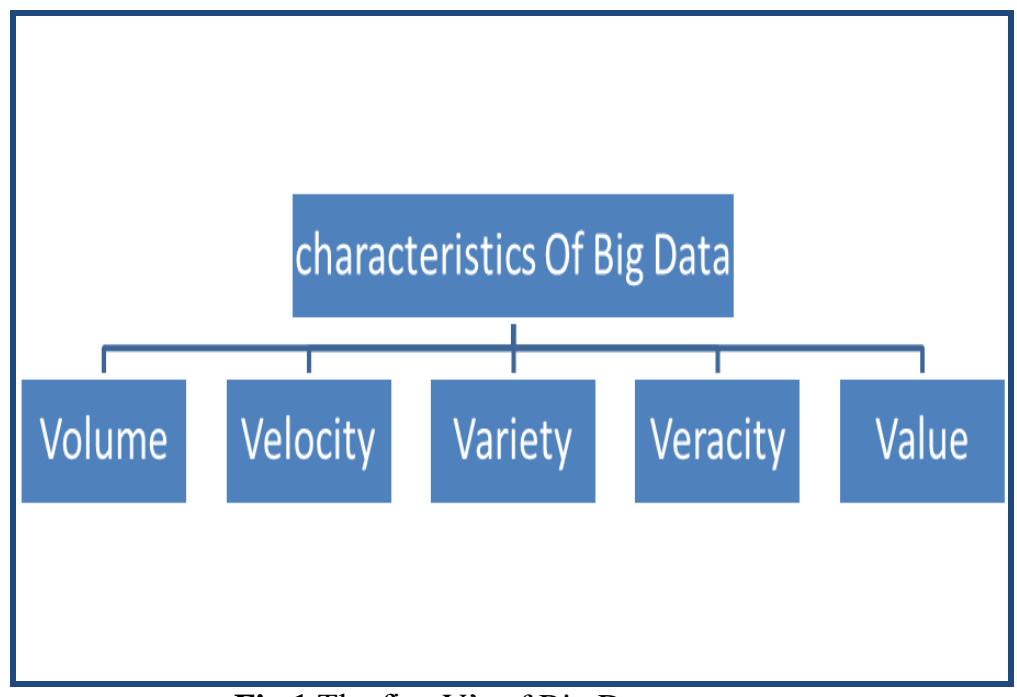

Fig.1 The five V's of Big Data

1) Volume: refers to large amounts of any kind of data from any different sources, including mobile digital data creation devices and digital devices. The benefit from gathering, processing, and analyzing these large amounts of data generates a number of challenges in obtaining valuable knowledge for people and companies $[11,12,13]$. The volume of data is on the increase with all the consequences on storage and processing.

2) Velocity: The second key dimension of Big Data is velocity [9] or the relentless rapidity of data creation. Marketing executives with access to rich, insightful, current data are able to make better decisions based on evidence at a given time, rather than on intuition or laboratory-based consumer research. To better appreciate the difference between large data sets and Big Data, consider the difference between U.S. census data and consumer data collected by a leading women's clothing retailer-whose marketing executive knows at any given time how many consumer transactions are occurring; which product, styles, and colors of merchandise are moving off store shelves as well as the retailer's website; and what consumers are posting on social networks about the retailer. Both types of data are rich, large, and provide insights. Only the latter, however, gives the marketing executive the ability to make current and evidence-based decisions that competitors without Big Data insight will be hard-pressed to match.

3) Variety: Variety means the increasing complex of data types. the data are not only traditional structured relational data e.g., ERP data, but also many semi-structured data e.g., the weather data, Web services data, emails and tweets, as well as unstructured data like customer behavior data and the audio and video data [14].

4) Value: refers to the process of extracting valuable information from large sets of social data, and it is usually referred to as Big Data analytics. Value is the most important characteristic of any Big Data based application, because it allows to generate useful business information $[11,12,13]$.

5) Veracity: refers to the correctness and accuracy of information. Behind any information management practice lie the core doctrines of data quality, data governance, and metadata management, along with considerations of privacy and legal concerns $[11,12,13]$.

Currently there are too many scenarios where the term Big Data appears. Scientists, business executives, practitioners of media and advertising and governments alike regularly meet difficulties with large data sets in areas including Internet search, finance and business informatics $[15,16]$.

The application of Big Data in modern business allows enterprises to achieve real competitive advantage, and enables research centers to increase the effectiveness of the conducted explorations. The analysis of large 
volumes of data is used in the following sectors: financial, telecommunications, health care, biotechnology, scientific research [2].

So, there is a vast opportunity offered by Big Data technologies to discover new insights that drive significant business value. Industries are seeing data as a market differentiator and have started reinventing themselves as "data companies", as they realize that information has become their biggest asset. Customer sentiment analysis through social media and call logs have given new insights into customer satisfaction. Network performance patterns have been analyzed to discover new ways to drive efficiencies. Customer usage patterns based on web click-stream data have driven innovation for new products and services to increase revenue [17].

\subsection{Related Research}

At the time of writing this paper, there has a few papers-reviewed published article found talking about «Big Data and ERP » or « «ERP and Big Data ».

According to the state of the art carried out by [18]. The results indicated that Big Data and ERP have been divorced in the literature. This is why the objective of the paper [18] is to draft a research agenda for future research, which combines both ERP and Big Data. $[18,19]$ explained how Big Data can create value-added to organizations:

- Making information transparent and usable at a higher frequency.

- As organizations create and store more and more transactional data in a digital form, they can collect more accurate and detailed performance information on everything from product inventories to sick days.

- It allows a fine-grained segmentation of customers and therefore much more precisely tailored products or services to meet their needs and requirements.

- Sophisticated analytics can substantially improve decision making quality.

- Big Data can also be used to improve the development of the next generation of products and services.

[18] suggested research, which combines both ERP and Big Data together forming a capable, and powerful technology platform such as:

- The integration between ERP and Big Data should enable organizations to have a better and wider-in-scope best practices. So research needs to be done in order to explore the impact on the organization.

- Organizations need the study the impact of Big Data on their regular IT infrastructure, with regards to the infrastructure supporting ERP systems.

- New techniques need to be studied and tested in order to preserve the data. In this context different data masking, encryption, etc. need to be tested for appropriateness.

- Big Data could be used to build a comprehensive view of different MDM elements e.g., customer. This poses a new challenge as well as an opportunity for ERP systems, which requires further research and exploration of impact on database, security, performance, etc.

- There is a need for the development of models and frameworks in order to explore the potentials of unstructured data and how they could be analyzed in a way that would further enrich the data stored in ERP systems.

- Integrating the outcome of Big Data into ERP systems would enable ERP systems to act as the knowledge hub, breaking the barrier of internal data only. But, that is easier said than done. Processing, storage and analytics remain as challenges which require further explorations.

- Marrying ERP data with Big Data requires where the confidence level must be established at raw as well as aggregation levels. This requires further investigation to the harmonization techniques and confidence measurements.

- One of the key strength of an ERP system is its functionality; it is the ability to enable, manage and monitor key business functionalities. Functionality of ERP systems has attracted lots of organizations, over the years, to adopt them. Now, with the introduction of Big Data, we believe that ERP functionality will never be the same again.

- The integration between ERP systems and Big Data has the potential to change the ERP lifecycle phases, or activities within e.g., during ERP selection organizations might rely on semi-structured as well as unstructured data, from social networks, to evaluate ERP alternatives.

Then, according to the state of the art done, we propose in the following a generic meta-model that shows the marriage of ERP and Big Data, to enrich transactional data used by traditional ERP with external data (from social networks, sensor, etc.). This new meta-model will improve the functionality offered by a conventional ERP and thus improve decision making and add value to the business.

\section{Our Methodological Approach}

Based on several ERP (Open source or commercial) we propose a fragment of the meta-model simplified, explicitly, whatever ERP is associated with a single transactional database (SQL statements) (Fig. 2). In the event, the ERP has multi-business, it is associated with several modules (CRM, SRM, WMS, MRP, TMS, 
etc.), each module with its proposed features while communicating with other modules to ensure integration system and share the same data. Each actor (internal business) queries with the appropriate module with transactional instructions (insert, delete, update and select). From a strategic perspective, each ERP system is distinguished by its dashboard based on the schema of the database to generate indicators of behavior and the states in the form of reports. The quality of indicators and reports is the data reliability and pertinence of the indicators.

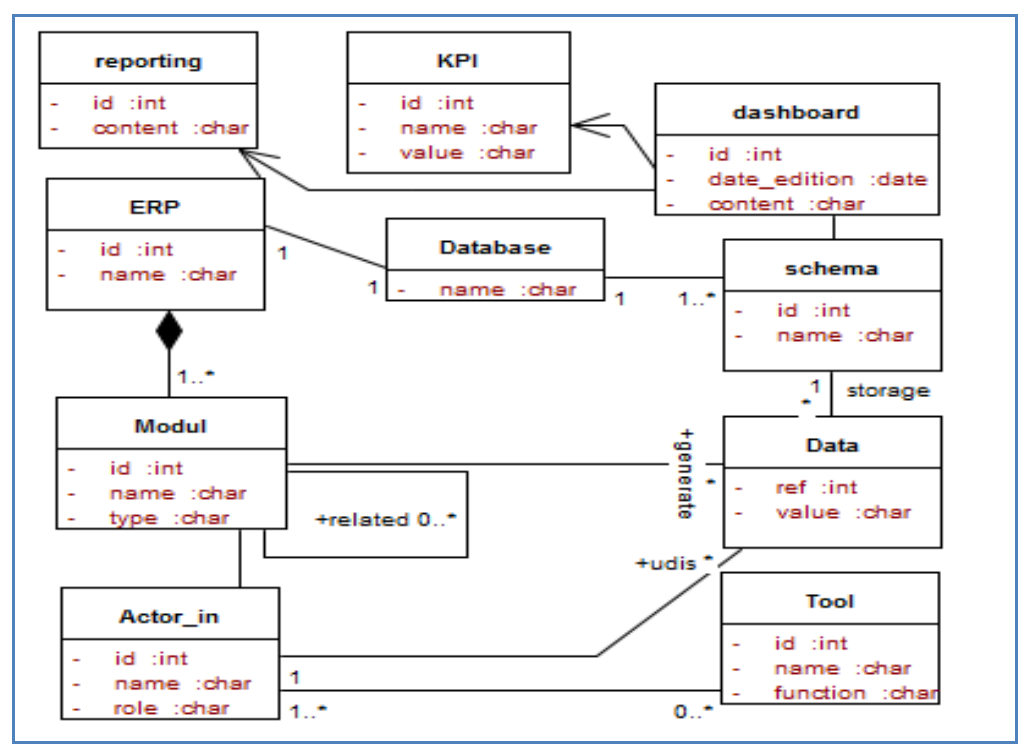

Fig.2 ERP meta-model with a single transactional database

According to the meta-model in Figure 2, we deduce that the generated indicators are calculated after a latency due to the validation of the internal actor by ignoring the state of the machine in real time.

According to the descriptions of Big Data, we propose the meta-model functioning schematically represented in Figure 3, the data is heterogeneous generated by internal actors (business) and external actors. On the branch of the internal actor, Big Data accepts transactional data and more, and according to the activities of each actor, Big Data collecting data in real time through the actor of the activity itself or through the tools used by the actor through sensors and according to the specificity of each business (RFID, GPS, signal, etc.). The external actor also represents a really interesting source of information to enrich the data (profile, social sites, assessments, etc.). About data, they are distinguished by a wide variety of types (txt, img, GPS, etc.). The challenge lies how to filter relevant information from the vast amount of information collected by Big Data. By following how do flirters aggregate information with transaction data from an ERP system?

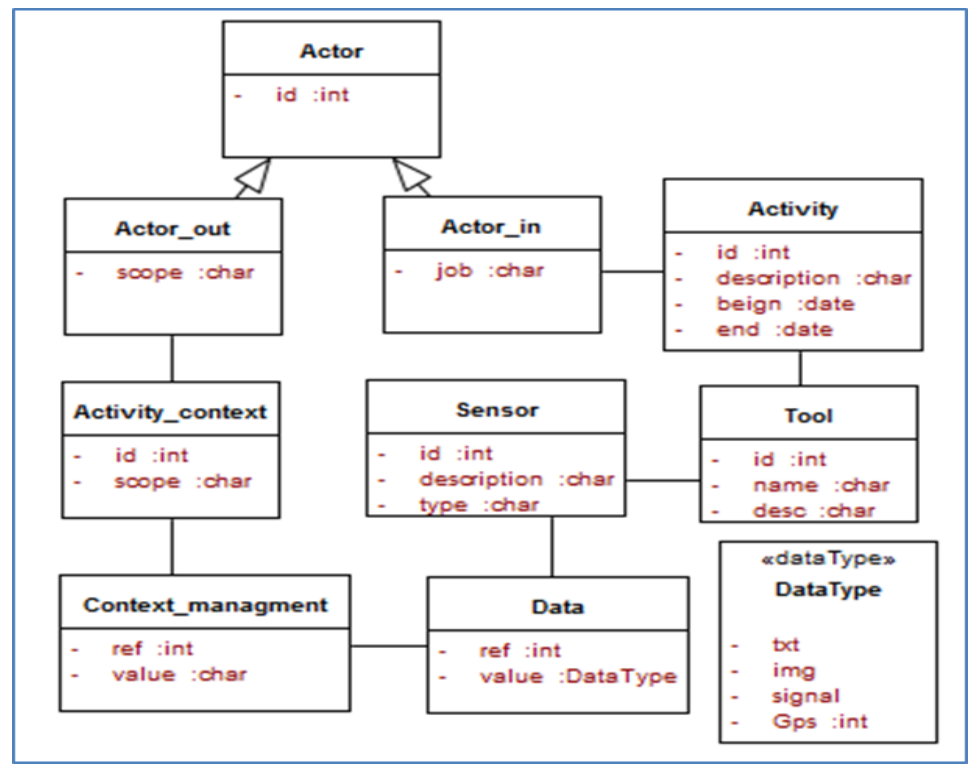

Fig.3 Big Data meta-model 
Good decisions are driven by the quality and reliability of information. In this vision, Big Data is a very interesting source for enriching and reliable ERP Data System, but it remains to know how to exploit the two data sources. In the Fig. 4, we propose our vision for the aggregation of data from the two systems, namely, the ERP generates data, we call Data1, and Big Data generates data, we call them Data2, the Date2 must pass a set filters to capture that information about a specific and determined appropriate level for the business concerned. Thereafter, we will get filtered data Data_filtre, according to a merge mechanism we perform the merger between the data and data_filtre Data1 to get Data_final will be more reliable and responsive.

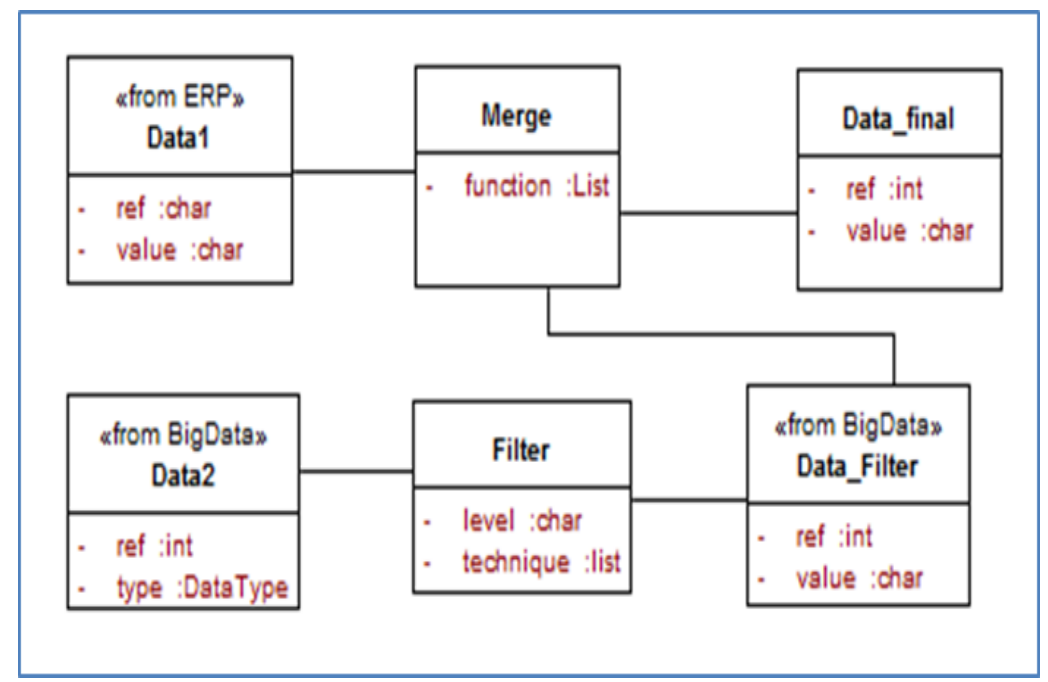

Fig. 4. Aggregation of data from Big Data and ERP

\section{Results and Discussion}

Based on ERP meta-model (Fig. 2), Big Data meta-model (Fig. 3) and Aggregation of data from Big Data and ERP (Fig. 4), we propose our generic meta-model of a new BIG ERP (Fig. 5).

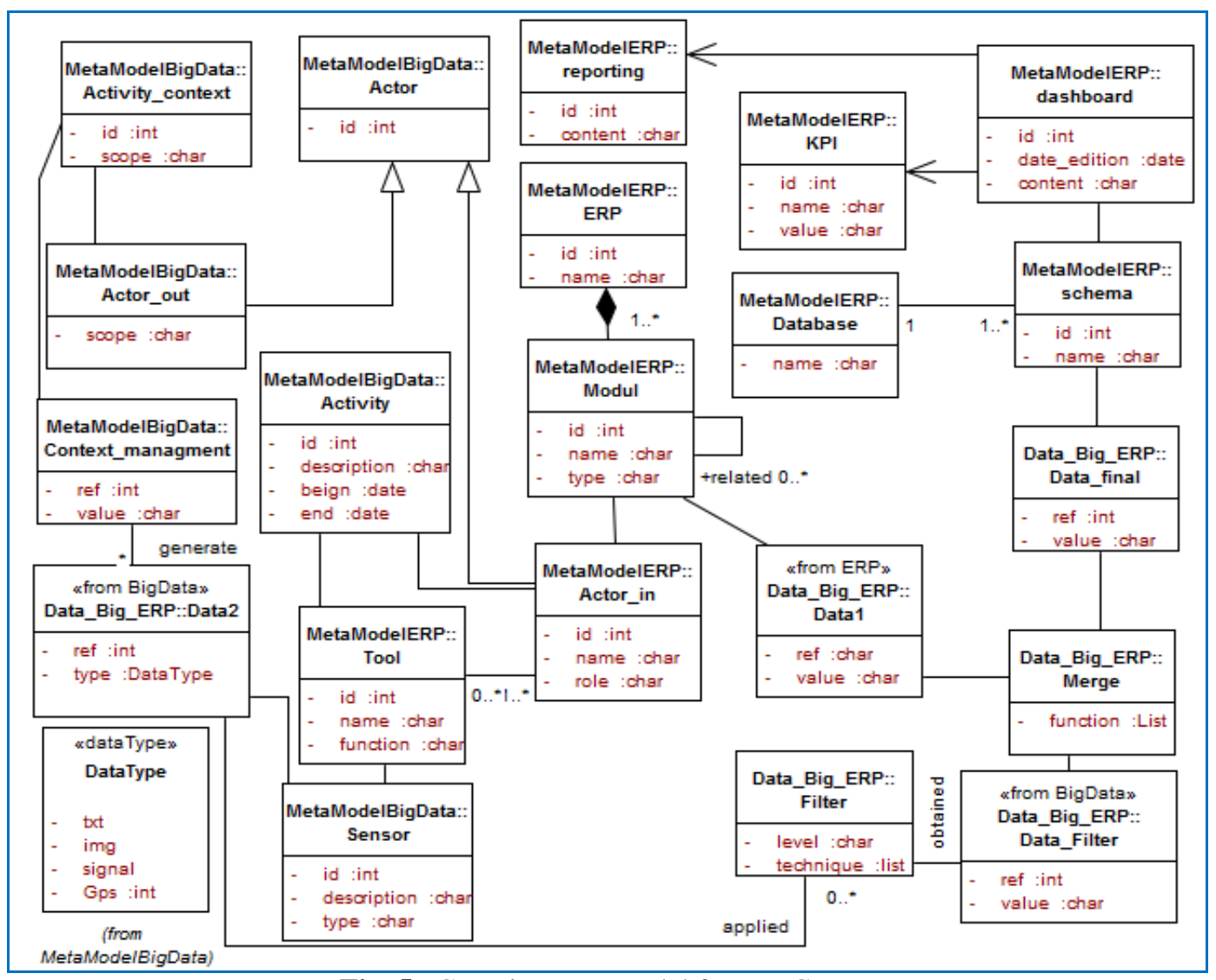

Fig. 5. Generic Meta-Model for a BIG ERP 
After presenting the meta-model ERP, Big Data and that which concerns the merger between the two systems, we present the meta-model result, this meta-model is composed of meta-classes from all three packages, namely: MetamodelERP, MetaModelBigData and Data_Big_ERP. Each meta-class that presents itself once in meta-models, it automatically added to the meta-model resulting. For example, meta-class module from MetamodelERP package and meta-class Activity from MetaModelBigData package. Nevertheless, for the metaclasses that figures at least in two meta-models, we keep a single occurrence of a single package after comparing attributes to each meta-classes, for example, the meta-class Actor_in. At last to distinguish the difference data between ERP system and Big Data, we labeled Data1 for ERP and Data2 for Big Data.

\section{Conclusion and Future Prospects}

We believe strongly that the future of ERP systems will definitely incorporate Big Data. Below is a suggested research which propose a generic meta-model that combines both ERP and Big Data together forming a capable, and powerful technology platform. First, we have proposed an ERP meta-model with a single transactional database. After that, we have proposed a Big Data meta-model. Finally, we have been presenting a generic meta-model that shows the marriage of ERP and Big Data, to enrich transactional data used by traditional ERP with external data (from social networks, sensor, etc.). This new meta-model will improve the functionality offered by a conventional ERP and thus improve decision making.

Future research should also address questions like: how to validate the proposed meta-model? How can we ensure the quality of data after the integration of data from Big Data and ERP?

\section{References}

[1]. Akkermans, H., \& Van Helden, K. (2002). Vicious and virtuous cycles in ERP implementation: A case study of interrelations between critical success factors. European Journal of Information Systems, 11(1), 35-46.

[2]. Helena Kościelniak (2015), Agnieszka Puto, BIG DATA in decision making processes of enterprises, International Conference on Communication, Management and Information Technology (ICCMIT 2015), (C) 2015 The Authors. Published by Elsevier B.V.

[3]. Klaus, H., Rosemann, M., \& Gable, G. G. (2000). What is ERP? Information Systems Frontiers, 2(2), 141-162.

[4]. Moon, Y. B. (2007). Enterprise resource planning (ERP): A review of the literature. International Journal of Management and Enterprise Development, 4(3), 235-264.

[5]. Hong, K. K., \& Kim, Y. G. (2002). The critical success factors for ERP implementation: An organizational fit perspective. Information and Management, 40(1), 25-40.

[6]. Kalling, T. (2003). ERP systems and the strategic management processes that lead to competitive advantage. Information Resources Management Journal, 16(4), 46-67.

[7]. David Gil 2016. Modeling and Management of Big Data: Challenges and opportunities. Future Generation Computer Systems 63 (2016) 96-99. (C) 2015 Elsevier B.V.

[8]. IBM (2012). What is big data? IBM Corporate Website (Retrieved May 24, 2012 from http://www01.ibm.com/software/data/bigdata/)

[9]. Lycett, M. (2013).'Datafication': Making sense of (big) data in a complex world. European Journal of Information Systems, 22(4), 381-386.

[10]. Oracle (2012). Big data for the enterprise. Oracle White Paper,1-14 (Retrieved February 1, 2014 from http://www.oracle.com/us/products/database/big-data-forenterprise-519135.pdf).

[11]. Gema Bello-Orgaz, Jason J. Jung, David Camacho, Social big data: Recent achievements and new challenges InformationFusion28(2016)45-59 @ 2015 Elsevier B.V

[12]. D. Laney, 3D Data Management: Controlling Data Volume, Velocity, and Variety, Technical Report, 2001.URLhttp://blogs.gartner.com/doug-laney/files/2012/01/ad949-3D-Data-Management-Controlling-Data-Volume-VelocityandVariety.pdf(accessed August 2015)

[13]. I.A.T. Hashema, I. Yaqooba, N.B. Anuara, S. Mokhtara, A. Gania, S.U. Khanb, The rise of big data on cloud computing: review and open research issues, Inf. Syst.47 (2015) 98-115.

[14]. Kaile Zhou, Chao Fu, Shanlin Yang (2015); Big data driven smart energy management: From big data to big insights; Renewable and Sustainable Energy Reviews 56 (2016) 215-225; 2015 Elsevier Ltd.

[15]. K. Cukier, Data, data everywhere: a special report on managing information, in: Economist Newspaper, 2010.

[16]. A. Labrinidis, H. Jagadish, Challenges and opportunities with big data, Proc.VLDB Endow. 5 (12) (2012) $2032-2033$.

[17]. Donna Burbank; http://enterprisearchitects.com/the-5v-s-of-big-data/.

[18]. Ahmed Elragal (2014); ERP and Big Data: The Inept Couple; CENTERIS 2014; Procedia Technology 16 (2014) 242 - 249 Elsevier.

[19]. Manyika, J., Chui, M., Brown, B., Bughin, J., Dobbs, R., Roxburgh, C., Byers, A.H. (2011), Big Data: The Next Frontier forInnovation, Competition, and Productivity. In McKinsey Global Institute Reports, pp.1-156. 